\title{
Bempedoic Acid a Small Molecule Drug Process and Synthesis, Innovation And /Or Advantages, Development Status And /Or Regulatory Status
}

\author{
Krishnasarma Pathy* \\ Head R\&D-QC/QA, IPL Research Centre, India \\ *Corresponding author: Krishnasarma Pathy, Head R\&D-QC/QA, IPL Research Centre, India
}

Received: 制 July 04, 2019

Published: 眥July 15, 2019

\begin{abstract}
Bempedoic acid (ETC-1002), a small molecule drug, promotes low density lipoprotein (LDL) receptor mediated clearance of LDL-cholesterol (LDL-C) by inhibition of adenosine triphosphate citrate lyase (ACL), a mechanism complementary to those of existing lipid-modifying therapies. Bempedoic acid is a pro-drug activated specifically within the liver where it inhibits ACL, a regulatory checkpoint within the cholesterol biosynthesis pathway. By inhibiting ACL, bempedoic acid reduces cholesterol synthesis in liver cells and triggers compensatory LDL receptor upregulation. Inhibiting ACL with bempedoic acid complements other mechanisms targeted by current therapies, resulting in additional lowering of LDL-C, without leading to increases in adverse events (AEs).b In the phase III clinical trials (NCT02666664, NCT02991118) of patients with high cardiovascular risk and elevated LDL-C not adequately controlled by their current therapy, patients are given a daily dose of $180 \mathrm{mg}$ bempedoic acid as an oral tablet, whilst remaining on ongoing lipid-modifying therapy. The present paper describes the process and synthesis, innovation and /or advantages, development status and /or regulatory status of Bempedoic acid.
\end{abstract}

Keywords: Cardiovascular Disease; Hyperlipidemia; ETC-1002; Low Density Lipoprotein Cholesterol; Synthesis; Statin Associated Muscle Symptoms; Statin Intolerance; Regulatory Status

\section{Introduction}

The current standard of care for patients with hypercholesterolaemia is primarily statins which can reduce LDL-C. However, some patients, particularly those with heterozygous familial hypercholesterolaemia, coronary heart disease (CHD), CHD-risk equivalents, and other clinical manifestations of atherosclerotic cardiovascular disease [1] (ASCVD), require additional LDL cholesterol lowering on top of what can be achieved with maximum tolerated statin therapy. Additionally, there are patients who are unable to tolerate statins due to adverse advents such as muscle pain, or increased blood glucose. There is an unmet medical need for patients unable to achieve sufficient reduction in LDL cholesterol with existing treatment options and thus remain at increased risk of cardiovascular disease and the consequences thereof. Bempedoic acid requires activation by a specific enzyme acyl-CoA synthatase (ACSVL1), which is largely restricted to the liver. Therefore, it is believed that unlike statins, myotoxicity is unlikely to occur with bempedoic acid because it does not inhibit cholesterol biosynthesis in skeletal muscle due to the absence of

ACSVL1 in these cells. The effect of bempedoic acid is additivenot redundant-to that of statins, because the target of bempedoic acid, ACL, is a distinct regulatory checkpoint on the cholesterol biosynthesis pathway than HMG-CoA reductase, the primary target of statins. Inability to tolerate statins because of muscle symptoms contributes to uncontrolled cholesterol levels and insufficient cardiovascular risk reduction. Bempedoic acid, a prodrug that is activated by a hepatic enzyme does not present in skeletal muscle, inhibits ATP-citrate lyase [2], an enzyme upstream of $\beta$ hydroxy $\beta$-methylglutaryl-coenzyme A reductase in the cholesterol biosynthesis pathway. Statins are widely prescribed for lowering LDL-cholesterol (LDL-C) and reducing the risk of cardiovascular disease. However, many patients are statin intolerant and unable to achieve sufficient LDL-C lowering due to muscle-related side effects. Bempedoic acid (ETC-1002)'s mechanism of action is similar to that of statins, but because it does not inhibit the cholesterol biosynthesis pathway in skeletal muscle, myotoxicity is unlikely to occur. ETC-1002 was first discovered at the original Esperion Therapeutics, which was acquired by Pfizer in 2004 and 
subsequently spun-out as Esperion Therapeutics in 2008 along with ETC-1002 and other assets. Esperion continues development of ETC-1002 [3], which is currently in phase III trials as an LDL-Clowering agent in patients with hypercholesterolemia. This review discusses the drug candidate's mechanism of action, effects, safety and clinical data. This paper explaining how the process of synthesis was carried and conclusion of Bempedoic acid offers a safe and effective oral therapeutic option for lipid lowering in patients who cannot tolerate statins.

a) The phase 3 CLEAR (Cholesterol Lowering via Bempedoic acid, an ACL-Inhibiting Regimen) Serenity clinical trial demonstrates the lipid-lowering efficacy of bempedoic acid, a firstin-class, prodrug, small-molecule inhibitor of ATP-citrate lyase, among patients with established statin intolerance and elevated low-density lipoprotein cholesterol who were receiving stable background therapy.

b) Muscle-related symptoms contributed to the history of statin intolerance for almost all patients.

c) Although bempedoic acid acts on the same cholesterol biosynthesis pathway as statins, the muscle-related adverse event rate in CLEAR Serenity with bempedoic acid, which is not activated in skeletal muscle, did not differ from placebo, even among patients who had experienced muscle-related symptoms while on statin therapy [4].

\section{Development Status And/or Regulatory Designations}

Bempedoicacid does notcurrently have Marketing Authorisation in the EU/UK for any indication. Bempedoic acid or bempedoic acid with ezetimibe in a fixed-dose combination are in phase III clinical trials for the treatment of primary hypercholesterolaemia [5] (heterozygous familial and nonfamilial) or mixed dyslipidaemia in patients who are statin-intolerant, or for whom a statin is contraindicated. Bempedoic acid monotherapy and bempedoic acid with ezetimibe in a fixed-dose combination is also in phase III clinical trials for the treatment of primary hypercholesterolaemia (heterozygous familial and non-familial) or mixed dyslipidaemia in patients unable to reach LDL-Cholesterol goals with the maximum tolerated dose as an adjunct to diet in combination with a statin or statin with other lipid lowering therapies. Esperion Therapeutics was founded in April 2008 by former executives of, and investors in, the original Esperion Therapeutics which was founded in July 1998 and was bought by Pfizer for $\$ 1.3$ billion in 2004 and then spun out in 2008. ETC-1002 was first discovered at the original Esperion [5], and Esperion subsequently acquired the rights to it from Pfizer in 2008. Esperion own the exclusive worldwide rights to ETC-1002.

\section{Preparation}

Bempedoic acid [5,6] was prepared by condensation of 1,5-dibromopentane (I) with ethyl isobutyrate (II) by means of LDA in THF in the presence of DMPU at $-78{ }^{\circ} \mathrm{C}$ to give ethyl 7-bromo-2,2-dimethylheptanoate (III), which is dimerized with tosylmethyl isocyanide (IV) in the presence of $\mathrm{NaH}$ and Bu4NI in DMSO to yield diethyl 8-isocyano-2, 2,14,14-tetramethyl-8-(tosyl) pentadecanedioate $(\mathrm{V})$. Reaction of intermediate $(\mathrm{V})$ with aqueous
HClin CH2Cl2 affords 2,2,14,14-tetramethyl-8-oxopentadecanedioic acid diethyl ester (VI), which is hydrolyzed with aqueous $\mathrm{KOH}$ in refluxing EtOH/H2O to provide the dicarboxylic acid ESP-15228 (VII) (1-4). Ketone (VII) is finally reduced by means of NaBH4 in $\mathrm{MeOH}(1)$. Scheme 1[6].

\section{7-Bromo-2,2-Dimethylheptanoic Acid Ethyl Ester 7-Bromo-2,2-dimethylheptanoic acid ethyl ester [5]}

Under argon atmosphere and cooling with an ice-bath, a solution of lithium diisopropylamide in THF (1.7 L, 2.0 M, 3.4 mol) was slowly dropped into a solution of 1,5-dibromopentane $(950 \mathrm{~g}$, $4.0 \mathrm{~mol}$ ) and ethyl isobutyrate $(396 \mathrm{~g}, 3.4 \mathrm{~mol})$ in THF (5 L) while keeping the temperature below $+5 \mathrm{DC}$. The reaction mixture was sti $\pi$ ed at room temperature for $20 \mathrm{~h}$ and quenched by slow addition of saturated ammonium chloride solution (3L). The resulting solution was divided into three 4-L portions. Each portion was diluted with saturated ammonium chloride solution (5L) and extracted with ethyl acetate (2 '2L). Each 4-L portion of ethyl acetate was washed with saturated sodium chloride solution (2L), $1 \mathrm{~N}$ hydrochloric acid (2L), saturated sodium chloride solution (2L), saturated sodium bicarbonate solution (2L), and saturated sodium chloride solution (2L). The three separate ethyl acetate layers were combined into a single 12-L portion, dried over magnesium sulfate, and concentrated in vacuo to give the crude material $(1.7 \mathrm{~L})$ which was purified by vacuum distillation. Two fractions were obtained: the first boiling at $88-104{ }^{\circ} \mathrm{C} / 0.6$ ton (184.2 g), the second at $105-120{ }^{\circ} \mathrm{C} / 1.4$ ton (409.6 g) for atotal yield of $60 \%$. 1H NMR (300 MHz, CDC1 1 TMS): $\delta$ (ppm): 4.11 (q, $2 \mathrm{H}, \mathrm{J}=7.2 \mathrm{~Hz}), 3.39$ (t, $2 \mathrm{H}, \mathrm{J}=6.8 \mathrm{~Hz}), 1.85$ $(\mathrm{m}, 2 \mathrm{H}), 1.56-1.35(\mathrm{~m}, 4 \mathrm{H}), 1.24(\mathrm{t}, 3 \mathrm{H}, \mathrm{J}=7.2 \mathrm{~Hz}), 1.31-1.19(\mathrm{~m}$, $2 \mathrm{H}), 1.16$ (s, $6 \mathrm{H}) \cdot{ }^{13} \mathrm{C} \mathrm{NMR}\left(75 \mathrm{MHz}, \mathrm{CDCI}_{3} / \mathrm{TMS}\right): \delta$ (ppm): 177.9, 60.2, 42.1, 40.5, 33.8, 32.6, 28.6, 25.2, 24.2, 14.3. HRMS (El, pos): Calcd. for $\mathrm{C} \pi \mathrm{H}_{22} \mathrm{Br} \theta 2\left(\mathrm{MH}^{+}\right)$: 265.0803, found: 265.0810.6.18.

\section{2,2,14,14-tetramethyl-8-oxo-pentadecanedioic acid diethyl ester}

Under Air atmosphere, to a solution of 7-bromo-2,2dimethylheptanoic acid ethyl ester ${ }^{5}$ (26.50 g, $\left.100 \mathrm{mmol}\right)$, tetra-nbutylammonium iodide (3.69 g, $10 \mathrm{mmol}$ ) and p- toluenesulfonyl methyl isocyanide ( $9.80 \mathrm{~g}, 50 \mathrm{mmol})$ in anhydrous DMSO (300 mL) was added sodium hydride (4.80 g, $20.5 \mathrm{mmol}, 60 \%$ dispersion in mineral oil) at $5-10^{\circ} \mathrm{C}$ The reaction mixture was sti $\pi$ ed at room temperature for $20 \mathrm{~h}$ and quenched with ice-water (300 $\mathrm{mL}$ ). The product was extracted with dichloromethane (3D 100 $\mathrm{mL})$. The combined organic layers were washed with water (200 $\mathrm{mL}$ ), half-saturated $\mathrm{NaCl}$ solution $100 \mathrm{~mL}$ ), and saturated $\mathrm{NaCl}$ solution (200 mL), dried over MgS04, and concentrated in vacuo to get the crude 8-isocyano-2,2,14,14-teframethyl-8-(toluene-4sulfonyl)-pentadecanedioic acid diethyl ester (36.8 g) as an orange oil, which was used in the next step without purification. To a solution of this crude product (36.8 g) in dichloromethane (450 $\mathrm{mL}$ ) was added concentrated hydrochloric acid $(110 \mathrm{~mL})$ and the mixture was stized at room temperature for $1 \mathrm{~h}$. The solution was diluted with water $(400 \mathrm{~mL})$ and the aqueous layer was extracted with dichloromethane $(200 \mathrm{~mL})$. The combined organic layers were washed with saturated NaHC0 solution ( $2 \times 150 \mathrm{~mL})$ and saturated 
$\mathrm{NaCl}$ solution (150 mL). The organic solution was dried over $\mathrm{Na} 2 \mathrm{~S} 04$ and concentrated in vacuo. The residue was subjected to column chromatography (silica gel, hexanes: ethyl acetate $=11: 1$ ) to give 2,2,14,14-tetramethyl-8-oxo-pentadecanedioic acid diethyl ester (12.20 g, $66 \%$ over two steps) as a colorless oil. 'H NMR (300 MHz, CDC1 $\left.1_{3} / \mathrm{TMS}\right): \delta(\mathrm{ppm}): 4.11(\mathrm{q}, 4 \mathrm{H}, \mathrm{J}-6.9 \mathrm{~Hz}), 2.37(\mathrm{t}, 4 \mathrm{H}$, J - 7.5 Hz), $1.58-1.47$ (m, 8 H), $1.35-1.10$ (m, 8 H), 1.24 (t, 6 H, J $=7.2 \mathrm{~Hz}), 1.15$ (s, $12 \mathrm{H}) \cdot{ }^{13} \mathrm{C}$ NMR $(75 \mathrm{MHz}, \mathrm{CDC} 13 / \mathrm{TMS}): \delta(\mathrm{ppm}):$ 211.6, 178.3, 60.5, 43.1, 42.5, 40.9, 30.1, 25.5, 25.1, 24.1, 14.7. HRMS (LSIMS, nba): Calcd. for $\mathrm{C}_{3} \mathrm{IL}_{3} \mathrm{O}_{5}\left(\mathrm{MH}^{+}\right)$: 399.3110, found: 399.3129 .

\section{8-OXO-2,2,14,14-Tetramethylpentadecanedioic Acid}

A solution of $\mathrm{KOH}(25 \mathrm{~g})$ in water $(50 \mathrm{~mL})$ was added to a solution of 2,2,14,14-tetramethyl-8-oxo-pentadecanedioic acid diethyl ester [6] (10.69 g, $155 \mathrm{mmol}$ ) in ethanol (400 mL), then heated at reflux for $4 \mathrm{~h}$. After cooling, the solution was evaporated to a volume of ca. $50 \mathrm{~mL}$ and diluted with water $(800 \mathrm{~mL})$. The organic impurities were removed by extracting with dichloromethane $(2 \mathrm{x}$ $200 \mathrm{~mL}$ ). The aqueous layer was acidified to $\mathrm{pH} 2$ with concentrated hydrochloric acid (50 mL) and extracted with methyl tert. -butyl ether (MTBE, 3 × $200 \mathrm{~mL}$ ). The combined organic layers were dried over magnesium sulfate and concentrated in vacuo to give the crude product $(9.51 \mathrm{~g}$ ) as an oil. Crystallization from hexanes / MTBE (50 mL: $25 \mathrm{~mL}$ ) afforded 8-oxo-2,2,14,14- teframethylpentadecanedioic acid (6.92 g, $79 \%$ ) as waxy, white crystals. M.p.: $83-84{ }^{\circ} \mathrm{C} .1 \mathrm{H} \mathrm{NMR}$ (300 MHz, $\left.\mathrm{CDCI}_{3} / \mathrm{TMS}\right): \delta(\mathrm{ppm}): 12.03(\mathrm{~s}, 2 \mathrm{H}), 2.37$ (t, $4 \mathrm{H}, \mathrm{J}=7.3$ $\mathrm{Hz}), 1.52-1.34(\mathrm{~m}, 8 \mathrm{H}), 1.28-1.10(\mathrm{~m}, 8 \mathrm{H}), 1.06(\mathrm{~s}, 12 \mathrm{H}) .{ }^{13} \mathrm{C} \mathrm{NMR}$ (75 MHz, $\mathrm{CDCI}_{3} / \mathrm{TMS}$ ): $\delta$ (ppm): 210.5, 178.8, 41.7, 41.2, 29.1, 25.0, 24.4, 23.1. HRMS (LSIMS, gly): Calcd. for $\mathrm{C}_{19} \mathrm{H}_{35} \mathrm{O}_{5}\left(\mathrm{MH}^{+}\right)$: 343.2484 , found: 343.2485 .

\section{8-Hydroxy-2.2.14,14-Tetramethylpentadecanedioic Acid}

Under nitrogen atmosphere, sodium borohydride $(0.06 \mathrm{~g}$, $1.6 \mathrm{mmol})$ was added to a stimed solution of 8-oxo-2,2,14,14tetramethylpentadecanedioic acid $(1.18 \mathrm{~g}, 3.4 \mathrm{mmol})$ in methanol (50 $\mathrm{mL}$ ) at $0{ }^{\circ} \mathrm{C}$. The reaction progress was momtored by thin layer chromatography (silica; hexanes: ethyl acetate $=50: 50$ ). Additional sodium borohydride was added after $1 \mathrm{~h}(0.48 \mathrm{~g}, 13 \mathrm{mmol})$. After $8 \mathrm{~h}$, the reaction mixture was hydrolyzed with water $(50 \mathrm{~mL})$ and acidified with concentrated hydrochloric acid (3 mL) to $\mathrm{pH} 1$. The solution was diluted with water $(50 \mathrm{~mL})$ and exfracted with dichloromethane (4 x $25 \mathrm{~mL}$ ). The combined organic layers were washed with saturated sodium chloride solution $(2 \times 30 \mathrm{~mL})$, dried over magnesium sulfate, concentrated in vacuo, and dried in high vacuo to give 8-hydroxy-2,2,14,14-tetramethylpentadecanedioic acid $(0.7 \mathrm{~g}, 60 \%)$ as a very viscous oil. ${ }^{1} \mathrm{H}$ NMR $(300 \mathrm{MHz}$, CDC1 $1_{3} / \mathrm{TMS}$ ): $\delta$ (ppm): 7.42 (br. s, $3 \mathrm{H}$ ), 3.59 (br. s, $1 \mathrm{H}$ ), 1.65 $1.00(\mathrm{~m}, 20 \mathrm{H}), 1.18$ (s, $12 \mathrm{H}) .{ }^{13} \mathrm{C}$ NMR (75 MHz, CDC1 $\left.1_{3} / \mathrm{TMS}\right): \delta$ (ppm): 184.5, 71.8, 42.1, 40.5, 37.0, 29.8, 25.2, 25.1, 24.9, 24.8. HRMS (FAB): Calcd. for $\mathrm{Cl}_{9} \mathrm{H}_{37} \mathrm{O}_{5}\left(\mathrm{MH}^{+}\right): 345.2635$, found: 345.2646 . HPLC: $83.8 \%$ purity. Keto-substituted hydrocarbons with 11-19 methylene and bis-terminal hydroxyl and carboxyl groups have been synthesized and evaluated in both in vivo and in vitro assays for their potential to favorably alter lipid disorders including metabolic syndrome. Compounds were assessed for their effects on the de novo incorporation of radiolabeled acetate into lipids in primary cultures of rat hepatocytes as well as for their effects on lipid and glycemic variables in obese female Zucker fatty rats [Crl:(ZUC)-faBR] following 1 and 2 weeks of oral administration. The most active compounds were found to be symmetrical with four to five methylene groups separating the central ketone functionality and the gem dimethyl or methyl/aryl substituents. Furthermore, biological activity was found to be greatest in both in vivo and in vitro assays for the tetramethyl-substituted keto diacids and diols (e.g., 10c, 10g,14c), and the least active were shown to be the bis(arylmethyl) derivatives (e.g., 10e, 10f,14f). Compound $14 \mathrm{c}$ dose-dependently elevated HDL-cholesterol, reduced triglycerides, and reduced NEFA, with a minimum effective dose of $30 \mathrm{mg} / \mathrm{kg} /$ day. Compound $10 \mathrm{~g}$ dose-dependently modified non-HDL-cholesterol, triglycerides, and no esterified fatty acids, with a minimum effective dose of $10 \mathrm{mg} / \mathrm{kg} /$ day. At this dose, compound 10g elevated HDL-cholesterol levels 2-3 times higher than pretreatment levels, and a dose-dependent reduction of fasting insulin and glucose levels was observed.

\section{Only Keto Compd Described}

\section{2,2,14,14-Tetramethyl-8-oxopentadecanedioic Acid [6] (10g).}

According to the procedure given for $\mathbf{1 0 f}, \mathbf{9 g}(8.54 \mathrm{~g}, 21.4 \mathrm{mmol})$ was saponified with $\mathrm{KOH}(85 \%, 4.53 \mathrm{~g}, 68.6 \mathrm{mmol})$ in $\mathrm{EtOH}(13 \mathrm{~mL})$ and water $(5 \mathrm{~mL})$ at reflux for $4 \mathrm{~h}$. The solid product obtained after usual workup was recrystallized from $\mathrm{Et}_{2} \mathrm{O} /$ hexanes $(50 \mathrm{~mL} / 50 \mathrm{~mL})$, affording $10 \mathrm{~g}(4.16 \mathrm{~g}, 57 \%)$ as colorlessneedles. Mp: $82-83{ }^{\circ} \mathrm{C} .{ }^{1} \mathrm{H}$ NMR (CDCl $)$ ): $\delta 11.53(\mathrm{br}, 2 \mathrm{H}), 2.39(\mathrm{t}, 4 \mathrm{H}, J=7.3), 1.60-1.50(\mathrm{~m}$, $8 \mathrm{H}), 1.30-1.20$ (m, $8 \mathrm{H}), 1.18(\mathrm{~s}, 12 \mathrm{H}) .{ }^{13} \mathrm{C} \mathrm{NMR}\left(\mathrm{CDCl}_{3}\right): \delta 211.7$, 185.0, 42.8, 42.3, 40.4, 29.7, 25.1, 24.8, 23.8.

HRMS (LSIMS, gly): calcd for $\mathrm{C}_{19} \mathrm{H}_{35} \mathrm{O}_{5}\left(\mathrm{MH}^{+}\right) 343.2484$, found 343.2444. HPLC: Alltima C-8 column, $250 \times 4.6 \mathrm{~mm}, 5 \mu \mathrm{m} ; 60 \%$ acetonitrile/40\% $0.05 \mathrm{M} \mathrm{KH}_{2} \mathrm{PO}_{4}$, flow rate $1.0 \mathrm{~mL} / \mathrm{min}$; $\mathrm{RI}, t_{\mathrm{R}} 6.50$ min, $92.6 \%$ pure. Bempedoic acid is an oral medicinal product that is in clinical development for the treatment of people with primary hypercholesterolaemia or mixed dyslipidaemia [7-9] with high cardiovascular risk. Abnormal levels of lipids in the blood characterises dyslipidaemia. High levels of cholesterol in the blood (hypercholesterolemia) may be caused by genetic defects as seen in familial hypercholesterolaemia or may occur when genes and other factors such as lifestyle habits interact, as seen in non-familial hypercholesterolaemia. Most people with hypercholesterolaemia have mildly or moderately increased low-density lipoprotein cholesterol (LDL-C) levels (often considered the "bad" cholesterol that may cause blockages of blood vessels). Elevated levels of LDL-C increase the risk of cardiovascular disease, which is responsible for many deaths and disabilities. Bempedoic acid lowers LDL-C via a different mechanism of action and offers the potential advantage of reduced muscular adverse effects when compared to statins which are the current standard of care. Bempedoic acid is being 
developed for patients at high cardiovascular risk who are unable to reach LDL-C goals with the maximum tolerated dose of statins. The effect of bempedoic acid is additive-not redundant-to that of statins, and if licensed, may offer additional and effective treatment option to use in combination with dietary changes and other lipidmodifying therapies to treat primary hypercholesterolaemia or mixed dyslipidaemia. Bempedoic acid is claimed in U.S. Patent No. $7,335,799$ that is scheduled to expire in December 2025, which includes 711 days of patent term adjustment, and might be eligible for a patent term extension period of up to five years. U.S. Patent Nos. 9,000,041, 8,497,301 and 9,624,152 claim methods of using bempedoic acid. PRODUCT: Bempedoic acid.

\section{Bempedoic Acid (Esperion Therapeutics, Inc.)}

With a targeted mechanism of action, bempedoic acid is a firstin-class, orally available, once-daily ACL inhibitor that reduces cholesterol biosynthesis and lowers elevated levels of LDL-C by up-regulating the LDL receptor, [10] but with reduced potential for muscle-related side effects. Completed Phase 1 and 2 studies in more than 800 patients treated with bempedoic acid have produced clinically relevant LDL-C lowering results of up to 30 percent as monotherapy, approximately 50 percent in combination with ezetimibe, and an incremental $20+$ percent when added to stable statin therapy.

\section{Mechanism of Action}

In November 2016, we announced the publication of "Liverspecific ATP-citrate lyase inhibition by bempedoic acid decreases LDL-C and attenuates atherosclerosis," by Stephen L. Pinkosky, our Associate Director of Translational Research and Biology, et al., in Nature Communications. The paper systematically outlines the experiments and analyses undertaken by us and our collaborators to fully understand the mechanism of action for how bempedoic acid reduces LDL-C, including its specificity for the liver. Bempedoic acid is a prodrug that once activated, inhibits ACL, an enzyme upstream of HMG-CoA reductase [11,12], (the molecular target of statins) in the cholesterol synthesis pathway. Like statins, bempedoic acid decreases cholesterol synthesis in the liver, which results in decreased intracellular cholesterol, up-regulation of LDL receptor activity and increased LDL-C clearance from the blood. Although bempedoic acid and statins both inhibit cholesterol synthesis in the liver, an important differentiating feature is that, unlike statins, bempedoic acid is inactive in skeletal muscle. Specifically, bempedoic acid is a prodrug which requires activation by a specific enzyme, very long-chain acyl-CoA synthetase, or ACSVL1, to convert bempedoic acid to its CoA activated form. This enzyme is present in the liver but not in skeletal muscle. Therefore, bempedoic acid does not inhibit the cholesterol biosynthesis pathway in skeletal muscle, thus providing a mechanistic basis for reduced potential for muscle-related adverse effects. Bempedoic acid has been shown to provide incremental lowering of LDL-C when used in combination with both ezetimibe and statins at all doses [13].

\section{Fixed Dose Combination Bempedoic Acid and Ezetimibe (BA+EZ)}

In the second quarter of 2016, the Food and DrugAdministration, or FDA, accepted our submission of an Investigational New Drug, or
IND, application for the fixed dose combination of bempedoic acid $180 \mathrm{mg}$ and ezetimibe $10 \mathrm{mg}$, or BA+EZ, which is in development for the same indications as bempedoic acid monotherapy $[14,15]$ (LDL-C lowering and CV risk reduction). We recently completed a bioavailability study and a formulation of BA+EZ has been selected for manufacturing, development and, if approved, commercialization. We expect to announce clinical development and regulatory plans for BA+EZ in the first half of 2017.

\section{Cardiovascular Disease and Elevated LDL-C}

Cardiovascular disease, which results in heart attacks, strokes and other cardiovascular events, represents the number one cause of death and disability in western societies. The American Heart Association, or AHA, estimates that approximately 800,000 deaths in the United States were caused by cardiovascular disease in 2013. Elevated LDL-C is well-accepted as a significant risk factor for cardiovascular disease and the CDC estimates that 78 million U.S. adults have elevated levels of LDL-C. A consequence of elevated LDL-C is atherosclerosis, which is a disease that is characterized by the deposition of excess cholesterol and other lipids in the walls of arteries as plaque. The development of atherosclerotic plaques often leads to cardiovascular disease. The risk relationship between elevated LDL-C and cardiovascular disease was first defined by the Framingham Heart Study, [15] which commenced in 1948 to define the factors that contributed to the development of cardiovascular disease. The study enrolled participants [16].

\section{(Esperion Therapeutics, Inc)}

\section{a) Licenses}

In April 2008, we entered into an agreement with Pfizer pursuant to which we acquired a worldwide, exclusive, fully paidup license from Pfizer to certain patent rights owned or controlled by Pfizer relating to bempedoic acid, and we granted Pfizer a worldwide, exclusive, fully paid-up license to certain patent rights owned or controlled by us relating to development programs other than bempedoic acid. The license to us covers the development, manufacture and commercialization of bempedoic acid. We may grant sublicenses under the license. Under the license agreement, Pfizer is restricted from making, using, developing or testing any of the compounds claimed under the same patents that claim or cover the composition of matter of bempedoic acid. [17], Neither party is entitled to any royalties, milestones or any similar development or commercialization payments under the license agreement, and the licenses granted are irrevocable and may not be terminated for any cause, including intentional breaches or breaches caused by gross negligence [18].

\section{Intellectual Property of Esperion Therapeutics, Inc.}

As of December 31, 2016, our patent estate, including patents we own or license from third parties, on a worldwide basis, included approximately 25 issued United States patents and four pending United States patent applications and 23 issued patents and 15 pending patent applications in other foreign jurisdictions. Of our worldwide patents and pending applications, only a subset relates to our small molecule program which includes our lead product candidate, bempedoic acid. Bempedoic acid is claimed in 
U.S. Patent No. 7,335,799 that is scheduled to expire in December 2025, which includes 711 days of patent term adjustment, and may be eligible for a patent term extension period of up to five years. U.S. Patent Nos. 9,000,041 and 8,497,301 claim methods of treatment using bempedoic acid. We also have a pending U.S. patent application directed to bempedoic acid. There are currently three issued patents and four pending application in countries outside the United States that relate to bempedoic acid [19].

\section{Overall Safety Observations (Esperion Therapeutics, Inc statement)}

To date, in completed studies, over 800 patients have been treated with bempedoic acid for periods of up to 12 weeks at maximum repeated doses of $240 \mathrm{mg}$ per day. Bempedoic acid has been safe and well-tolerated with no dose-limiting side effects identified to date in our ongoing or completed clinical studies. No clinical safety trends have emerged to date.

\section{Conclusion}

The synthesis of Bempedoic acid was prepared by condensation of 1,5-dibromopentane with ethyl isobutyrate by means of LDA in THF in the presence of DMPU at $-78{ }^{\circ} \mathrm{C}$ to give ethyl 7-bromo-2,2-dimethylheptanoate, which is dimerized with tosylmethyl isocyanide in the presence of $\mathrm{NaH}$ and $\mathrm{Bu} 4 \mathrm{NI}$ in DMSO to yield diethyl 8-isocyano-2, 2,14,14-tetramethyl-8-(tosyl) pentadecanedioate. Reaction of intermediate with aqueous $\mathrm{HCl}$ in $\mathrm{CH}_{2} \mathrm{Cl}_{2}$ affords 2,2,14,14-tetramethyl-8-oxopentadecanedioic acid diethyl ester, which is hydrolysed with aqueous $\mathrm{KOH}$ in refluxing EtOH/H2O to provide the dicarboxylic acid ESP-15228 (1-4) Ketone is finally reduced by means of $\mathrm{NaBH} 4$ in $\mathrm{MeOH}$ (1). Esperion announced new details about its phase 3 program for bempedoic acid, its unique oral, once-daily cholesterol-lowering compound. The company said the phase 3 program would include patients with hypercholesterolemia on any statin at any dose, including those with LDL levels not adequately controlled on current statin therapy in intolerant" patients unable to take even low doses of statins. Bempedoic acid does not currently have Marketing Authorisation in the EU/UK for any indication. Bempedoic acid or bempedoic acid with ezetimibe in a fixed-dose combination are in phase III clinical trials for the treatment of primary hypercholesterolaemia (heterozygous familial and nonfamilial) or mixed dyslipidaemia in patients who are statin-intolerant, or for whom a statin is contraindicated. Bempedoic acid monotherapy and bempedoic acid with ezetimibe in a fixed-dose combination is also in phase III clinical trials for the treatment of primary hypercholesterolaemia (heterozygous familial and non-familial) or mixed dyslipidaemia in patients unable to reach LDL- Cholesterol goals with the maximum tolerated dose as an adjunct to diet in combination with a statin or statin with other lipid lowering therapies.

\section{References}

1. Mancini GB, Baker S, Bergeron J, Fitchett D, Frohlich J, et al. (2016) Diagnosis, prevention, and management of statin adverse effects and intolerance: Canadian Consensus Working Group update (2016). Can J Cardiol 32: 35-65.
2. Rosenson RS, Baker S, Banach M, Borow KM, Braun LT, et al. (2017) Optimizing cholesterol treatment in patients with muscle complaints. J Am Coll Cardiol. 70: 1290-1301.

3. Stroes ES, Thompson PD, Corsini A, Vladutiu GD, Raal FJ, et al. (2015) Statin-associated muscle symptoms: impact on statin therapy-European Atherosclerosis Society Consensus Panel Statement on Assessment, Aetiology and Management. Eur Heart J 36: 1012-1022.

4. Cohen JD, Brinton EA, Ito MK, Jacobson TA (2012) Understanding Statin Use in America and Gaps in Patient Education (USAGE): an internetbased survey of 10,138 current and former statin users. J Clin Lipidol 6: 208-215.

5. Oniciu, DC, Dasseux JLH (Esperion Therapeutics, Inc.) Hydroxyl compounds and compositions for cholesterol management and related uses. WO 2004067489.

6. Oniciu, DC, Dasseux JLH (Esperion Therapeutics, Inc.) Ketone compounds and compositions for cholesterol management and related uses. US 2004198814.

7. Mueller R, Yang J, Duan C, Emil Pop, Otto J Geoffroy et al. (2004) Long hydrocarbon chain keto diols and diacids that favorably alter lipid disorders in vivo. J Med Chem 47(24): 6082-6099.

8. Ketone compounds and compositions for cholesterol management and related uses. WO 2005068412. Newton RS.

9. (2013) The Esperion story: Innovating in both good and bad economic times. Energy Metabolism \& Physiology Seminars for Healthy Living McMaster University, Hamilton.

10. Our history. Esperion Therapeutics Web site.

11. Shaffer C (2008) Pfizer jettisons Esperion. Nat Biotechnol 26(7): 724725 .

12. Cramer CT, Goetz B, Hopson KL, Fici GJ, Ackermann RM, et al. (2004) Effects of a novel dual lipid synthesis inhibitor and its potential utility in treating dyslipidemia and metabolic syndrome. J Lipid Res 45(7): 12891301.

13. Srivastava RA, Pinkosky SL, Filippov S, Hanselman JC, Cramer CT, et al. (2012) AMP-activated protein kinase: an emerging drug target to regulate imbalances in lipid and carbo-hydrate metabolism to treat cardio-metabolic diseases. J Lipid Res 53(12): 2490-2514.

14. Pinkosky SL, Filippov S, Srivastava RA, Hanselman JC, Bradshaw CD, et al. (2013) AMP-activated protein kinase and ATP-citrate lyase are two distinct molecular targets for ETC-1002, a novel small molecule regulator of lipid and carbohydrate metabolism. J Lipid Res 54(1): 134151.

15. Filippov S, Pinkosky SL, Newton RS (2014) LDL-cholesterol reduction in patients with hypercholesterolemia by modulation of adenosine triphosphate-citrate lyase and adenosine monophosphate-activated protein kinase. Curr Opin Lipidol 25(4): 309-315.

16. Pinkosky SL, Newton RS, Day EA, Ford RJ, Lhotak S, et al. (2016) Liverspecific ATP-citrate lyase inhibition by bempedoic acid decreases LDL-C and attenuates atherosclerosis. Nat Commun 7: 13457.

17. Bilen O, Ballantyne CM (2016) Bempedoic acid (ETC-1002): an investigational inhibitor of ATP citrate lyase. Curr Atheroscler Rep 18(10): 61 .

18. Banach M, Stulc T, Dent R, Toth PP (2016) Statin non-adherence and residual cardiovascular risk: there is need for substantial improvement. Int J Cardiol 225: 184-196.

19. Laufs U, Filipiak KJ, Gouni Berthold I, Catapano AL, SAMS Expert Working Group (2017) Practical aspects in the management of statin-associated muscle symptoms (SAMS). Atheroscler Suppl 26: 45-55. 
(c) (i) This work is licensed under Creative

To Submit Your Article Click Here: Submit Article

DOI: $10.32474 /$ SCSOAJ.2019.03.000154

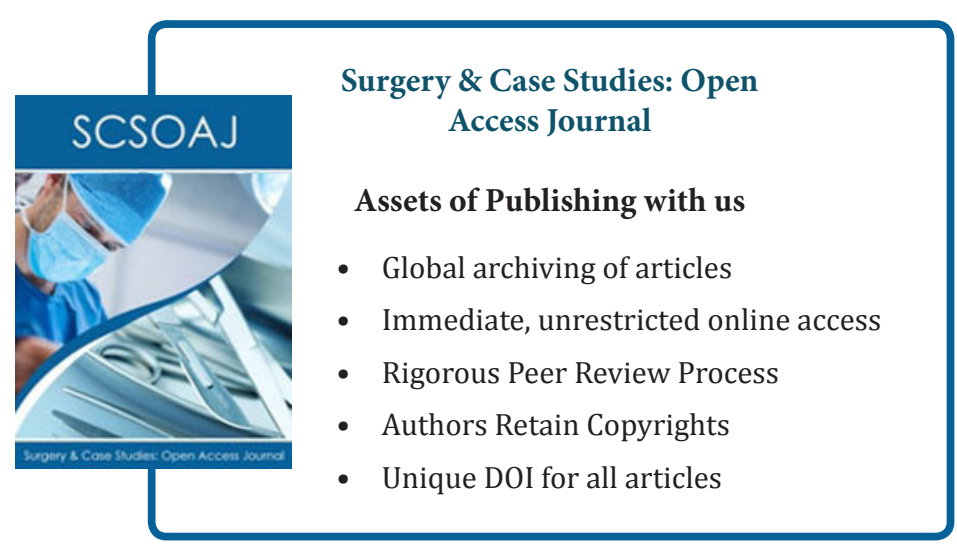

